\title{
Accrescimento o coerenza: il se' nei contesti sociali
}

\author{
R. KODILJA (1) y L. ARCURI (2)
}

(1) Dipartimento di Psicologia dello Sviluppo e della Socializzazione - Padova.

(2) Istituto di Psicologia - Trieste.

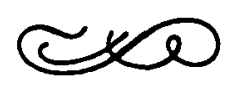

\section{Riassunto}

In questo lavoro viene valutata l'incidenza dei fattori interpersonali e situazionali nella rievocazione dei tratti di personalita riferiti a sé. La dimensione interpersonale presa in considerazione si riferisce ai motivi di auto-accrescimento e coerenza del sé che guidano la codifica delle informazioni riferite a sé. I soggetti, suddivisi in due gruppi (quelli con prevalente motivo di accrescimento e quelli con prevalente motivo di coerenza) vengono assegnati ad una delle tre condizioni sperimentali (accrescimento, coerenza e controllo). I risultati indicano che nella situazione di accrescimento, rispetto alle altre due condizioni sperimentali, $i$ soggetti utilizzano un maggior numero di tratti positivi per auto-descriversi. In un successivo compito di rievocazione, la condizione di accrescimento produce la prestazioni migliori per $i$ tratti positivi e quelle peggiori per $i$ tratti negativi.

Palabras clave: identidad, contexto.

\section{Enhancement or Consistency: The Self in Social Contexts}

\section{Abstract}

In this investigation we examinated the impact of individual differences and social context on the recall of self-referenced trait adjectives. Motives of self-enhancement and self-consistency, that determine the encoding of self-referenced information, have been considered as indices of individual difference. We assigned subject who possessed either prevalent need of self-enbancement or prevalent need of self-consistency to one of three different experimental conditions (enhancement, consistency and control). The tendency for subject to describe themselves with positive trait adjectives was particularly pronunced in the self-enbancement condition. Result in recall task indicate that best memory performances for positive adjectives and worse performances for negative adjectives were obtained both in the self-enhancement condition.

Keywords: self, social context.

Agradecimientos: Questo lavoro é stato effettuato nell'ambito dell'attività del Dottorato di Ricerca in Psicologia Sociale e dello Sviluppo del primo autore. E' stato reso possibile grazie ai finanziamenti MPI $40 \%$ del secondo autore.

Dirección del autor: Luciano Arcuri, Dipartimento di Psicologia dello Sviluppo e della Socializzazione, via Beato Pellegrino, 26 - PADOVA. 


\section{INTRODUZIONE}

Recentemente, gli psicologi di orientamento cognitivista hanno dedicato molta attenzione allo studio del concetto di sé, inserendo tale costrutto nel più ampio settore dei modelli schematici. Entro questo quadro teorico, il concetto di sé è rappresentato come un "sistema di schemi di sé» (Markus e Sentis, 1982), ossia un'articolata struttura di conoscenza sviluppata dagli individui e capace di integrare in maniera sistematica la grande quantità di informazioni riferite al sé. Attivando tali strutture le persone riuscirebbero a rappresentare e interpretare le proprie esperienze sociali. Secondo la definizione di Markus (1977), gli schemi di sé contengono astrazioni e generalizzazioni concernenti il proprio sé elaborate sulla base dei processi di categorizzazione e di valutazione messi in atto dall'individuo il quale, inoltre, raccoglie informazioni estrapolandole dal giudizio degli altri. $\mathrm{E}^{\prime}$ abbastanza implausibile che una rappresentazione mentale di sé contenga l'infinita quantità di informazioni riferibili a se stessi; è possibile invece che siano le informazioni che di volta in volta servono a mettere in evidenza le caratteristiche cruciali o salienti nel confronto tra sé e gli altri a ricoprire un ruolo centrale in questa organizzazione (Mc Guire e Padawer-Singer, 1976).

\section{L'effetto del riferimento a sé}

$\mathrm{Nel}$ vasto panorama delle ricerche empiriche sul ruolo delle strutture semantiche nell'elaborazione dell'informazione riferita al sé, sono largamente presenti gli studi sul recupero mnestico, da cui emerge che gli stimoli elaborati con un riferimento a sé vengono ricordati in misura superiore rispetto a qualsiasi altro tipo di materiale (Rogers, Kuiper e Kirker, 1977). Il fenomeno, comunemente definito come effetto del riferimento a sé (self-reference effect-SRE) sembra essere un effetto mnestico particolarmente robusto (vedi Greenwald, 1981 e Greenwald e Pratkanis, 1984 per una rassegna delle relazioni sé/memoria).

L'ormai classico paradigma sperimentale di Rogers, Kuiper e Kirker (1977) prevede un compito in cui i soggetti utilizzano alcuni tratti di personalità per descrivere se stessi, accanto ad altre condizioni nelle quali devono valutare le caratteristiche strutturali, fonemiche o semantiche dello stesso materiale. Il ricordo migliore, nei termini di quantità di aggettivi correttamente riconosciuti, si ottiene sempre nella condizione di riferimento a sé. La robustezza dell'effetto di riferimento a sé sembra confermato da ripetute verifiche empiriche (tra gli altri Kuiper e Rogers, 1979; Keenan e Baillet, 1980; Bower e Gilligan, 1979; Lord, 1980; Mc Caul e Maki, 1984, Bellezza, 1984; Klein e Kihlstrom, 1986), benché la sua interpretazione, a livello di modello teorico, rimanga tuttora molto controversa (Greenwald e Pratkanis, 1984).

A questo proposito, alcuni autori (Rogers, 1981; Kuiper, 1981) spiegano il fenomeno chiamando in causa un processo di confronto tra l'informazione in ingresso e lo schema di sé; altri (Bellezza, 1984) fanno riferimento all'attivazione di indizi interni che verrebbero impiegati in fase di recupero mnestico. Comunque, l'interpretazione dell'effetto SRE che gode di maggior fama (Rogers et. al., 1977; Bower e Gilligan, 1979, Keenan 
e Baillet, 1980) chiama in causa il processo di codifica delle informazioni: il compito di riferimento a sé provocherebbe una codifica del materiale molto più elaborata e profonda rispetto ad un compito neutro.

Le verifiche sperimentali dell'effetto SRE finora condotte evidenziano significative differenze nella rievocazione del materiale a seconda che questo sia riferito a sé oppure ad altri individui; inoltre emergono differenze di prestazione in rapporto al grado di familiarità del soggetto con le persone/target.

I risultati sperimentali hanno anche consentito di ricavare preziosi suggerimenti sulle caratteristiche del sé in quanto struttura cognitiva, sulle modalità di organizzazione in memoria del materiale auto-riferito nonché sul funzionamento di una grossa porzione di memoria, ovvero della memoria autobiografica.

\section{Le differenze interpersonali}

Bisogna comunque rilevare come in questo filone di ricerca sia stato assolutamente trascurato lo studio delle differenze interindividuali. In altre parole; non ci si è posti il problema di quale ruolo giochino fattori individuali (come le dimensioni motivazionali) nel direzionare il processo.

In questa prospettiva, un possibile criterio per individuare le differenze tra gli individui si riferisce al modo in cui due diversi principi o motivi guidano la selezione e codifica delle informazioni riguardanti la propria persona. Tali principi sono stati identificati nella:

I) Coerenza del sé («self-consistency»).

II) Auto-accrescimento («self-enhancement»).

Un motivo di coerenza di sé porterebbe l'individuo a selezionare e codificare i dati congruenti con l'immagine che egli già possiede di se stesso; questi andrebbero ad integrarsi a informazioni affini preesistenti nello schema di sé. D'altra parte secondo il principio di auto-accrescimento l'individuo tenderebbe a codificare quelle informazioni disponibili nell'ambiente che gli consentono di migliorare l'immagine di sé e quindi di incrementare sentimenti di soddisfazione ed efficacia personale. I teorici della coerenza di sé (a partire da Lecky, 1945; Secord e Backman, 1965; Swann, 1983) sostengono che il sistema cognitivo "preferisce» prendere in carico gli stimoli altamente prevedibili, familiari, stabili, che presentano la caratteristica di ridurre l'incertezza. I teorici dell'autoaccrescimento, introducendo quale variabile interveniente l'autostima soggettiva, ipotizzano che gli individui con un concetto di sé negativo ed un basso livello di auto-stima tendono a superare questa condizione privilegiando le informazioni che consentono di migliorare l'immagine di sé (Epstein, 1983; Rosenberg, 1968; Morse e Gergen, 1970).

Regan (1976) trova un punto di contatto tra i due filoni teorici. Egli ipotizza che nel caso in cui un giudizio riguardi una caratteristica di personalità o dominio comportamentale rispetto al quale l'individuo non possiede uno schema cognitivo viene attivato il meccanismo di incremento dell'auto-stima e miglioramento dell'immagine di sé, come previsto dalla teoria dell'auto-accrescimento; nel momento in cui il giudizio si riferisce ad una caratteristica per la quale l'individuo possiede uno schema già ben articolato interviene il meccanismo di coerenza del sé. 


\section{Le contingenze situazionali}

Alla luce di questi risultati emerge che questi due motivi sono copresenti negli individui in misura diversa in rapporto alla loro auto-immagine. $\mathrm{E}^{\prime}$ possibile, inoltre, che l'attivazione di uno o dell'altro principi dipenda dalle contingenze situazionali. Sarebbero quindi le caratteristiche del contesto, ovvero le richieste di coerenza o di accrescimento insite in una situazione specifica, ad accentuare il bisogno di coerenza o di accrescimento presenti nell'individuo.

L'attuale letteratura sulla «social cognition» sostiene che sono molteplici i fattori che incidono sul processo di elaborazione delle informazioni sociali: non solo le caratteristiche proprie dello stimolo, ma anche gli schemi di credenze e di aspettativa del soggetto, i motivi che lo guidano in un dato momento. Un'altro elemento molto importante è rappresentato dalle caratteristiche di contesto della situazione sociale entro cui l'individuo si muove (Higgins e McCann, 1984): infatti in relazione a tale situazione e sulla base degli obiettivi individuati, il soggetto mette in atto strategie di elaborazione appropriate e successivamente pianifica un comportamento efficace per rispondere in maniera adeguata alle esigenze della situazione (Showers e Cantor, 1985). La flessibilità di comportamento guidata dalle caratteristiche situazionali sembra essere la regola piuttosto che l'eccezione (vedi tra gli altri, Snyder, 1979; Gergen, 1979).

E' possibile che gli individui codifichino le conoscenze relative alla flessibilità del proprio comportamento nei termini di una gerarchia di concetti di sé contesto-specifici; ognuna di queste auto-immagini rappresenterebbe cioè l'impressione ricavata dal proprio comportamento in una delle diverse categorie di situazioni (Kihlstrom e Cantor, 1984; Kihlstrom, Cantor, Albright, Chew, Klein, Neidenthal, 1988). Come suggeriscono alcuni autori (McGuire e McGuire, 1981; McGuire, McGuire e Cheever, 1986; Markus e Nurius, 1986) sono proprio i fattori di tipo situazionale che hanno il potere di determinare quale aspetto della propria personalità sia opportuno mettere in evidenza e su quale caratteristica focalizzare la propria attenzione in un dato momento. E' l'individuo stesso, guidato dai propri scopi, a stabilire quale sia la dimensione di personalità più appropriata alla situazione. Sulla base di una distinzione degli scopi, Showers e Cantor (1985) differenziano gli "scopi rilevanti per il sé» in globali, astratti, quelli impiegati in varie situazioni, quelli concreti e quelli contesto-specifici. La loro funzione primaria sarebbe il mantenimento di un'elevato livello di autostima oppure di una coerenza nella concezione di sé; di conseguenza, le strategie cognitive messe in atto cambiano in rapporto alle contingenze situazionali (Carver e Scheier, 1981; Mischel, 1984). Al contrario Markus e Nurius (1986) e Markus e Wurf (1987) sottolineano la distinzione tra i diversi sé, differenziandoli tra sé correnti e sé possibili individuando nel "working self-concept» un concetto derivato dalla combinazione dei due precedenti.

\section{IPOTESI}

A partire dai dati di ricerca concernenti il ruolo dei motivi nell'elaborazione delle informazioni rigurdanti il sé ci siamo proposti di 
studiare le possibili relazioni tra caratteristiche situazionali e le modalità di orientamento del sé. In particolare abbiamo valutato:

a) In quale misura gli orientamenti del sé in fase di codifica (auto-accrescimento e coerenza del sé) influenzano l'auto-descrizione e la successiva rievocazione dei tratti di personalità;

b) quanto una situazione, che per le sue caratteristiche intrinseche favorisce l'attivazione del meccanismo di auto-accrescimento o di coerenza del sé, riesce a determinare la scelta dell'immagine da presentare in fase di auto-descrizione e ad influenzare la rievocazione del materiale.

1. Riferendoci ai dati di Brown (1986) che ha messo in luce l'esistenza di un bias di auto-accrescimento nel giudizio sociale, per cui in un compito di descrizione di sé e degli altri, gli individui descrivono con attributi positivi se stessi mentre attribuiscono agli altri soprattutto tratti di tipo negativo, abbiamo formulato le seguenti ipotesi:

a) Gli individui guidati da un prevalente bisogno di auto-accrescimento (d'ora in poi, SE) utilizzeranno un maggior numero di aggettivi positivi (socialmente desiderabili) nella fase di descrizione di sé rispetto agli individui con prevalente motivo di coerenza del sé (SC).

b) Sulla base del paradigna della profondità di elaborazione, il quale afferma che il grado di ritenzione del materiale è funzione del livello di elaborazione dello stimolo al momento della codifica, siamo partiti dall'assunzione che gli SC prestino molta attenzione a tutto il materiale, facilitando così una modalità di codifica più profonda rispetto agli $\mathrm{SE}$, i quali invece sarebbero più attenti alle caratteristiche di desiderabilità del materiale, codificandolo in maniera differenziata. Si presume quindi che i soggetti SE rievochino correttamente in misura maggiore gli aggettivi desiderabili, contrariamente agli SC, i quali dovrebbero ricordare in egual misura tutto il materiale.

2. Abbiamo formulato le seguenti ipotesi per quanto riguarda il ruolo del contesto situazionale:

a) Sulla base dei dati di ricerca si può assumere che, sia in fase di auto-descrizione, sia in fase di recupero mnestico, attraverso la mediazione di processi di codifica differenziati, i soggetti siano sensibili ai contesti situazionali del compito. Una situazione che richiede un'auto-presentazione finalizzata all'acquisizione di valutazioni positive da parte degli altri dovrebbe favorire la scelta da parte del soggetto di item prevalemtemente positivi quali elementi di auto-presentazione. Quando il compito di auto-descrizione non comporta la presentazione ipotetica ad altri, $i$ soggetti dovrebbero prediligere una immagine di sé meno polarizzata in relazione alla desiderabilità sociale dei tratti scelti. 
b) Anche in fase di recupero mnestico la presentazione dovrebbe risentire delle modalità di codifica differenziali che hanno caratterizzato l'acquisizione del materiale.

c) Tali effetti dovrebbero risultare amplificati dalle interazioni con le modalità di orientamento prevalente presente nei soggetti intervistati.

\section{METODO}

\section{Soggetti}

Ad un gruppo di 248 soggetti del nord-est d'Italia, di età compresa tra i 20 e 25 anni, é stato somministrato un questionario allo scopo di identificare l'orientamento prevalente del loro sé (Forzi, 1987). Tale strumento consente di indivuiduare sulla base delle risposte dei soggetti le relazioni esistenti tra i due motivi di auto-accrescimento e di coerenza del sé presenti in ciascun individuo.

Sono stati quindi selezionati 60 soggetti, sulla base di un'indice di rapporto tra il punteggio di coerenza e quello di accrescimento (raporto $=$ coerenza/accrescimento) $:$ i 30 soggeti con prevalente motivo di auto-accrescimento (SE) ed i 30 soggetti con prevalente motivo di coerenza del sé (SC) sono stati quindi casualmente assegnati ad una delle 3 condizioni sperimentali.

\section{Materiale e procedura}

\section{Fase}

Sono state scelte tre diverse situazioni sperimentali capaci di favorire rispettivamente:

1. Una codifica del materiale secondo il meccanismo dell'auto-accrescimento (condizione di accrescimento). Da una lista di 50 aggettivi i soggetti dovevano selezionarne almeno 10 per descrivere se stessi ad un possibile datore di lavoro. Gli aggettivi avrebbero dovuto fornire così un profilo di personalità in funzione di un'ipotetica assunzione.

2. Una codifica del materiale secondo il meccanismo di coerenza di sé (condizione di coerenza). In questo caso i soggetti erano posti di fronte ad uno specchio e contemporaneamente ripresi da una telecamera: la loro immagine era proiettata su uno schermo collocato davanti a loro. Il compito richiedeva un'auto-descrizione che il soggetto doveva proporre a se stesso: una sorta di diario in cui gli aggettivi scelti avrebbero dovuto rispecchiare i tratti di personalità che l'individuo riteneva di possedere.

3. Una codifica neutrale (condizione di controllo). La medesima lista di aggettivi veniva utilizzata dai soggetti per descrivere se stessi; nelle istruzioni non veniva specificata la finalità per cui tale auto-descrizione era richiesta. 


\section{Tebella I}

I 50 aggettivi (2S positivi e 25 negativi) utilizzati nel compito di auto-descrizione e relativi valori medi di positività e negatività

\begin{tabular}{|c|c|c|c|}
\hline Aggettivo & $\begin{array}{l}\text { Valore di } \\
\text { positività }\end{array}$ & Aggettivo & $\begin{array}{l}\text { Valore di } \\
\text { positività }\end{array}$ \\
\hline $\begin{array}{l}\text { affettuoso } \\
\text { allegro } \\
\text { altruista } \\
\text { attivo } \\
\text { competente } \\
\text { cortese } \\
\text { creativo } \\
\text { afficiente } \\
\text { fantasioso } \\
\text { generoso } \\
\text { gentile } \\
\text { indipendente } \\
\text { ingegnoso }\end{array}$ & $\begin{array}{l}1.9 \\
2.04 \\
2.28 \\
2.1 \\
1.68 \\
1.77 \\
2.33 \\
1.50 \\
1.69 \\
2.32 \\
1.95 \\
1.89 \\
2.12\end{array}$ & $\begin{array}{l}\text { intraprendente } \\
\text { leale } \\
\text { ordinato } \\
\text { paziente } \\
\text { puntuale } \\
\text { responsabile } \\
\text { schietto } \\
\text { sensibile } \\
\text { socievole } \\
\text { tenace } \\
\text { tollerante } \\
\text { tranquillo }\end{array}$ & $\begin{array}{l}1.62 \\
2.62 \\
1.18 \\
1.63 \\
1.55 \\
1.94 \\
2.32 \\
2.11 \\
2.26 \\
1.93 \\
1.25 \\
0.93\end{array}$ \\
\hline Aggetivo & $\begin{array}{l}\text { Valore di } \\
\text { negatività }\end{array}$ & Aggetivo & $\begin{array}{l}\text { Valore di } \\
\text { negatività }\end{array}$ \\
\hline $\begin{array}{l}\text { apatico } \\
\text { credulone } \\
\text { conformista } \\
\text { diffidente } \\
\text { egocentrico } \\
\text { egoista } \\
\text { facilone } \\
\text { geloso } \\
\text { goffo } \\
\text { incoerente } \\
\text { indeciso } \\
\text { insicuro } \\
\text { invidioso }\end{array}$ & $\begin{array}{l}-1.84 \\
-1.36 \\
-1.24 \\
-1.3 \\
-1.53 \\
-2.25 \\
-1.25 \\
-1.22 \\
-1.04 \\
-1.69 \\
-1.13 \\
-1.3 \\
-2.41\end{array}$ & $\begin{array}{l}\text { lagnoso } \\
\text { lunatico } \\
\text { noioso } \\
\text { pauroso } \\
\text { permaloso } \\
\text { pigro } \\
\text { possessivo } \\
\text { sboccato } \\
\text { scontroso } \\
\text { sdolcinato } \\
\text { superstizioso } \\
\text { vanitoso }\end{array}$ & $\begin{array}{l}-1.99 \\
-1.1 \\
-2.13 \\
-1.33 \\
-1.72 \\
-1.18 \\
-1.1 \\
-1.73 \\
-1.2 \\
-2.01 \\
-1.9 \\
-2.09\end{array}$ \\
\hline
\end{tabular}

I 50 aggettivi impiegati ( 25 positivi e 25 negativi) sono stati selezionati sulla base di un precedente studio di taratura (Colpo, 1976) su una lista di tratti di Anderson (1974; vedi Tabella I) in funzione del loro grado di desiderabilità sociale.

\section{Fase}

Dopo un intervallo di 48 ore i soggetti erano sottoposti ad una prova di memoria; posti nella medesima situazione di laboratorio dovevano rievocare il maggior numero possibile di aggettivi indipendentemente dal fatto che fossero stati impiegati oppure no nella fase di descrizione di sé.

\section{RISULTATI}

Sono stati calcolati ed utilizzati complessivamente 8 indici di rapporto, quali indicatori delle scelte fatte dai soggetti nel compito di auto-descrizione e delle prestazioni di memoria:

RP. Proporzione di aggettivi positivi sul totale degli aggettivi utilizzati nell'auto-descrizione. 
RN. Proporzione di aggettivi negativi sul totale degli aggetrivi utilizzati nell'auto-descrizione.

RMP. Proporzione degli aggettivi positivi correttamente rievocati sul totale degli aggettivi rievocati.

RMN. Proporzione degli aggettivi negativi correttamente rievocati sul totale di aggettivi rievocati.

RMDP. Proporzione degli aggettivi positivi rievocati, precedentemente utilizzati nell'auto-descrizione sul totale degli aggettivi rievocati e impiegati nell'auto-descrizione.

RMDN. Proporzione degli aggettivi negativi rievocati, precedentemente utilizzati nell'auto-descrizione sul totale degli aggettivi rievocati e impiegati nell'auto-descrizione.

RMLP. Proporzione degli aggettivi positivi rievocati non utilizzati precedentemente nell'auto-descrizione sul totale degli aggettivi rievocati non utilizzati nell'auto-descrizione.

RMLN. Proporzione degli aggettivi negativi rievocati non utilizzati precedentemente nell'auto-descrizione sul totale degli aggettivi rievocati non utilizzati nell'auto-descrizione.

Per chiarezza espositiva operiamo una distinzione tra la presentazione dei risultati ottenuti nella I fase (auto-descrizione) e quella relativa ai risultati del compito di rievocazione.

\section{Auto-descrizione}

E' stata effettuata un'analisi della varianza (ANOVA con il programma SPSS-X) secondo un disegno $3 \times 2$ (condizioni $X$ gruppo) considerando come variabile dipendente la proporzione di aggettivi positivi utilizzati. Si evidenzia un effetto significativo rispetto alla condizione sperimentale nel senso previsto dall'ipotesi $(F=2.98 ; p<.05)$; infatti nella situazione di auto-accrescimento (ACCR) viene impiegata una quantità significativamente superiore di aggettivi positivi $(\mathrm{M}=.77)$ rispetto alla situazione di coerenza del sé (COER: $\mathrm{M}=.64$; condizione di controllo: $M=.75)$. Un effetto significativo, ma nel senso inverso rispetto alle previsioni, viene registrato anche in rapporto ai due gruppi di soggetti. Gli individui guidati dal bisogno di coerenza del sé utilizzano un maggior numero di aggettivi positivi nell'auto-descrizione, soprattutto nella condizione di accrescimento $(M=.92)$, rispetto agli individui con prevalente motivo di accrescimento posti nella medesima condizione sperimentale $(\mathrm{M}=.63 ; \mathrm{F}=8.12, \mathrm{p}<.05)$ (fig. 1).

\section{Prestazioni di memoria}

Sono state effettuate 6 separate analisi della varianza per ciascun indice di memoria preso in considerazione (RMP, RMN, RMDP, RMDN, RMLP, RMLN). E' stata inoltre fatta un'analisi della varianza multivariata (MANOVA) per i 6 indici quali variabili dipendenti. In tutte le analisi condotte il disegno è stato $3 \times 2$ (condizione a 3 livelli: ACCR, COER, CONT e gruppo a 2 livelli: SE e SC).

Nel caso degli aggettivi positivi (RMP) si evidenzi un effetto significativo rispetto alla condizione sperimentale. Viene infatti rievocata una maggiore 


\section{9}

Figura 1

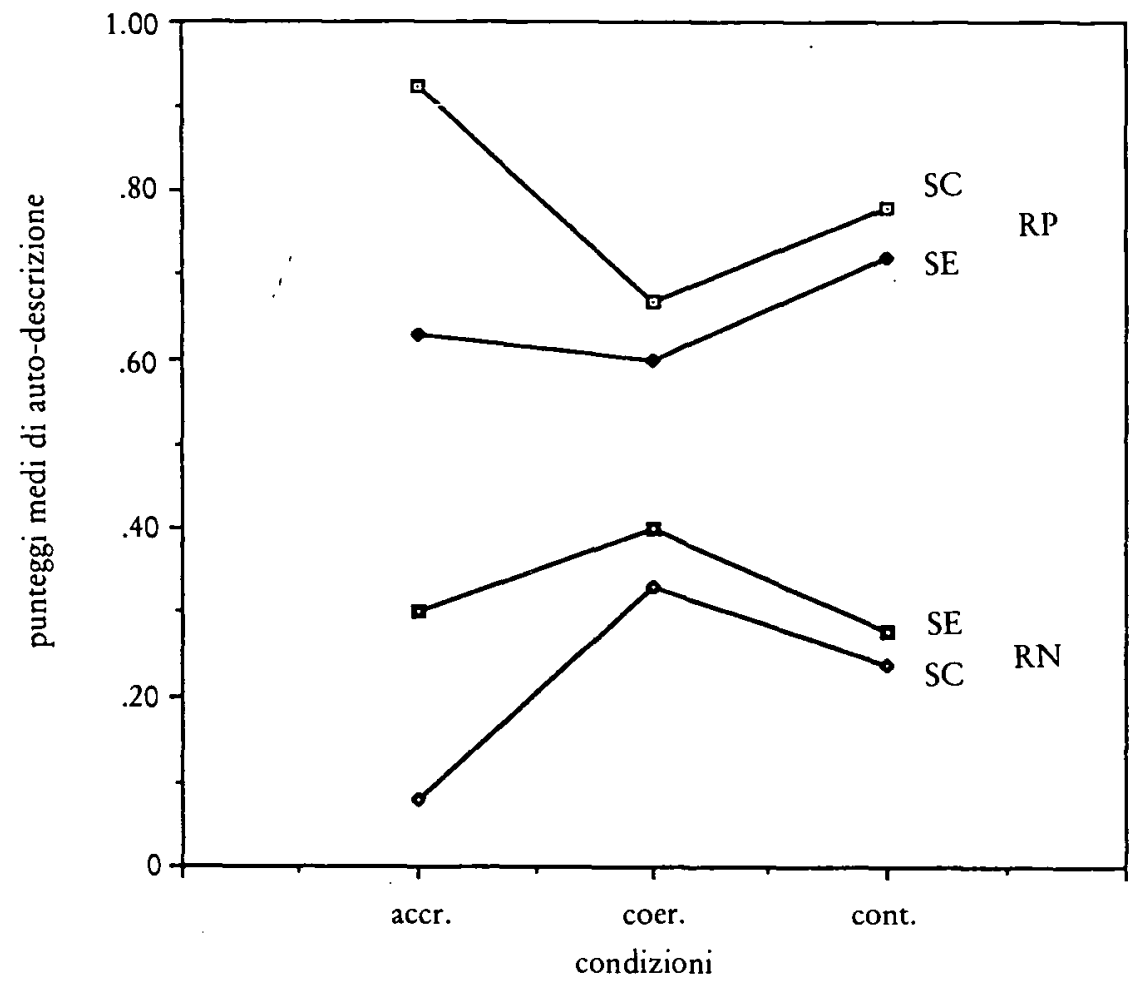

Medie degli aggettivi positivi $(R P)$ e negativi ( $R N)$ utilizzati nell'auto-descrizione rispetto alle 3 condizioni e per $i 2$ gruppi di soggetti (SC e SE).

quantità di aggettivi positivi nella condizione di accrescimento del sé $(\mathrm{M}=.70)$ rispetto alle rimanenti due situazioni (COER: $\mathrm{M}=.59$; CONT: $\mathrm{M}=.56 ; \mathrm{F}=3.71, \mathrm{p}<.05$ ). Questo pattern di risultati viene replicato anche per l'indice RMDP che indica la quantità di aggettivi positivi correttamente rievocati, precedentemente impiegati nell'auto-descrizione. L'effetto più significativo anche in questo caso è quello riferito alla condizione; infatti sono i soggetti posti nella condizione di accrescimento (soprattutto quelli guidati dal bisogno di coerenza) a rievocare più accuratamente (ACCR: $\mathrm{M}=.80$; COER: $\mathrm{M}=.60$; CONT: $\mathrm{M}=.72$; $\mathrm{F}=3.80, \mathrm{p}<.05)$.

Per tutti gli indici di rievocazione degli aggettivi negativi (RMN, RMDN, RMLN) si registrano effetti significativi rispetto alla situazione sperimentale. Infatti una situazione che richiede l'attivazione del meccanismo di coerenza del sé provoca sia una migliore rievocazione degli aggettivi negativi in generale (indice $R M N$, COER: $M=.41$; ACCR: $\mathrm{M}=.30 ;$ CONT: $\mathrm{M}=.44 ; \mathrm{F}=3.72, \mathrm{p}<.05)$ che una migliore rievocazione degli aggettivi negativi utilizzati nella auto-descrizione (indice RMDN, COER: $M=.40 ;$ ACCR: $M=.20$; CONT: $M=.28 ; F=3.80$, $\mathrm{p}<.05)$. Si rileva inoltre una differenza significativa nelle prestazioni di memoria per gli aggettivi negativi tra i due gruppi di soggetti: sono i soggetti 
Figura 2

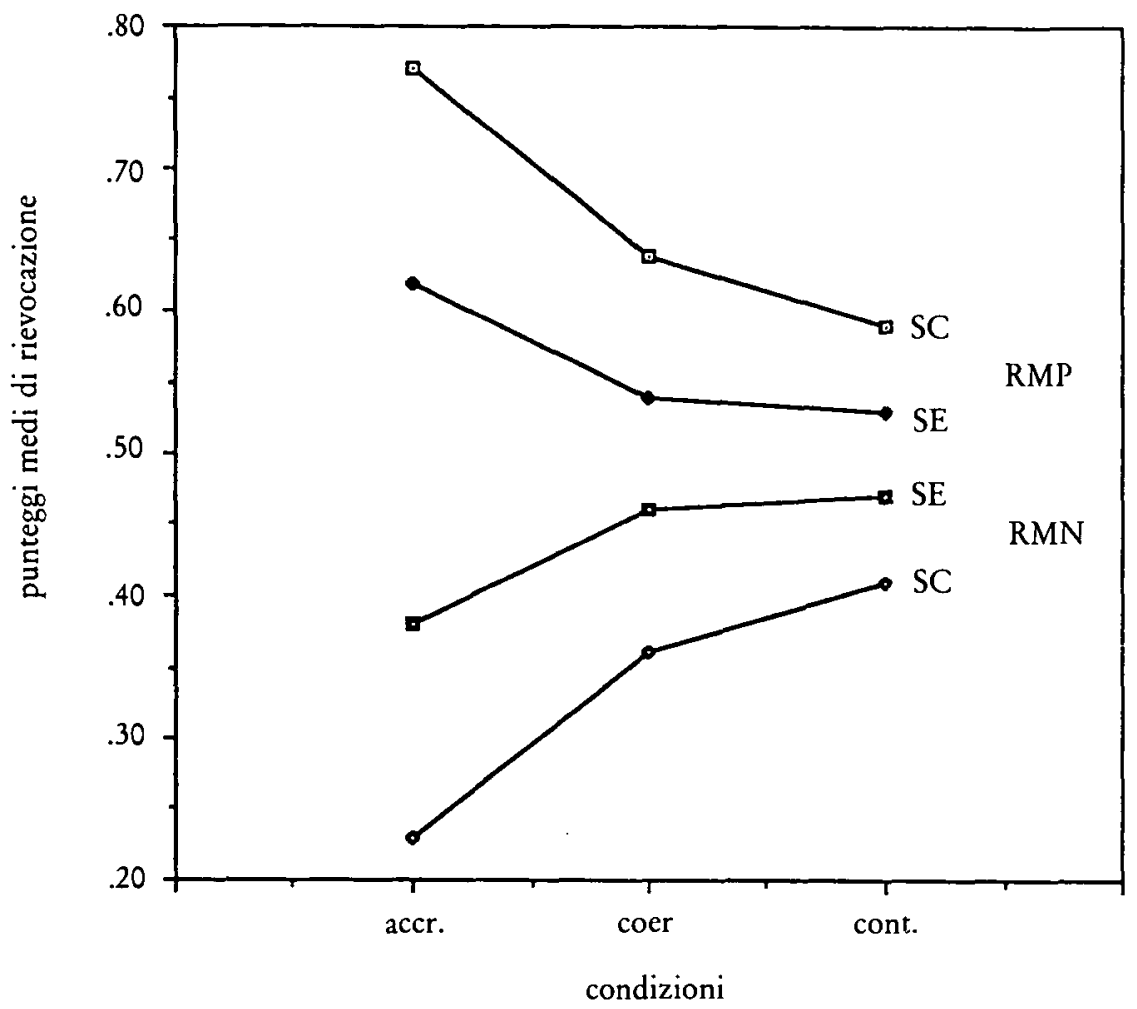

Medie degli aggettivi positivi (RMP) e negativi (RMN) correttamente rievocati nelle 3 condizioni, per $i$ due gruppi di soggetti (SC e SE).

SE $(\mathrm{M}=.44)$, rispetto agli SC $(\mathrm{M}=.33)$, ad evidenziare le prestazioni migliori $(\mathrm{F}=5.88, \mathrm{p}<.05)$.

Un'analisi della varianza multivariata rivela infine che non ci sono effetti interattivi tra la variabile "gruppo" e la variabile «condizione sperimentale» nel compito di rievocazione degli aggettivi positivi e negativi (fig. 2).

Un'analisi discriminante che si proponeva di confrontare i gruppi delle tre condizioni sperimentali impiegando quali variabili predittive gli indici di auto-descrizione e di rievocazione ha permesso di individuare due funzioni canoniche discriminanti. Tali funzioni anche se sono composte unicamente dalla variabile RN e dalla variabile RMNL consentono di discriminare significativamente le tre diverse condizioni. Le funzioni discriminano efficacemente la condizione di accrescimento dalla condizione di coeranza $\left(\mathrm{F}_{(2,54)}=3.95, \mathrm{p}<.05\right)$ e la condizione di accrescimento dalla condizione di controllo $\left(\mathrm{F}_{(2,54)}=3.31, \mathrm{p}<.05\right)$ e la condizione di coeranza da quella di controllo $\left(\mathrm{F}_{(2,54)}=3.34, \mathrm{p}<.05\right)$.

\section{DISCUSSIONE}

Il ricordo di tratti di personalità riferiti a sé sembra essere determinato in misura significativa dal "contesto sociale» mentre l'orientamento 


\section{1}

prevalente del sé sembra non incidere sulla prestazione di memoria nel senso previsto.

I risultati indicano, infatti, che una condizione di accrescimento dell'immagine di sé, capace di favorire una scelta di aggettivi positivi, ed una condizione di coerenza di sé, capace di favorire la scelta sia di aggettivi positivi che negativi, influenzano la rievocazione come ipotizzato. Le migliori prestazioni di memoria per gli aggettivi positivi si ottengono nella situazione di accrescimento, mentre nella situazione di coerenza diminuisce la quantità di aggettivi positivi correttamente rievocati. Nel caso degli aggettivi negativi si registra l'andamento opposto: le prestazioni peggiori si hanno per l'auto-accrescimento, ma migliorano nettamente nella situazione di coerenza. Ciò vale soprattutto per quegli aggettivi che erano stati precedentemente considerati come auto-descrittivi.

L'incidenza delle caratteristiche interpersonali ha fatto invece registrare un andamento inverso rispetto a quello previsto. I soggetti guidati da un prevalente motivo di accrescimento ricordano un maggior numero di aggettivi negativi; mentre, i soggetti con il bisogno di mantenere una stabile immagine di sé e che quindi dovrebbero rievocare in egual misura tratti negativi e positivi, ricordano una maggiore quantità di aggettivi positivi. L'effetto di accrescimento del sé, attribuibile ad una motivazione soggettiva, già evidenziato nei compiti di auto ed etero-valutazione (Moreland e Sweneey, 1984; Brown, 1986; Swann et al., 1987) e nei compiti di memoria per i tratti di personalità (Forzi, Kodilja, Arcuri, 1988), non viene riscontrato in questo contesto. $\mathrm{E}^{\prime}$ interessante però notare che già Shrauger nel 1975 e successivamente Moreland e Sweeney (1984) e Swann et al. (1987) constatano che le "reazioni di tipo cognitivo" dei soggetti sono influenzate dai bisogni di coerenza del sé mentre le «reazioni di natura affettiva» sono influenzate dal bisogno di auto-accrescimento. Se questa distinzione fosse valida anche rispetto al tipo di prestazione richiesta ai soggetti ed assumendo che un compito di rievocazione possa essere considerato come compito prettamente cognitivo, si potrebbe fornire una spiegazione dei risultati in questi termini.

Un'ulteriore problema sorge nel momento in cui prendiamo in considerazione $\mathrm{i}$ dati registrati nella fase di auto-descrizione. La prevista tendenza degli individui guidati dall'auto-accrescimento a descriversi in termini significativamente più positivi rispetto agli individui guidati dalla coerenza, non viene riscontrata empiricamente. Si verifica anzi l'effetto opposto: sono le persone con un bisogno di coerenza di sé ad utilizzare un maggior numero di aggettivi positivi ed un minor numero di aggettivi negativi per l'auto-descrizione rispetto all'altro gruppo di soggetti. Quindi, neanche in un compito di auto-descrizione viene registrato un'effetto di accrescimento dell'immagine di sé.

Alla luce di questi dati, le differenze interindividuali sulla dimensione accrescimento/coerenza sembrano essere inconsistenti, mentre si nota una preponderanza dei fattori dovuti al contesto. L'orientamento del sé più che dipendere da caratteristiche individuali sembra essere indotto dalla situazione condizionata al tipo de compito. Il set situazionale non limita la sua influenza alla fase operante (descrizione) ma incide successivamente anche sulla rievocazione.

Un ricco filone di studi identifica negli scopi (goals) di natura 
motivazionale gli elementi che portano a sviluppare adeguate strategie di elaborazione delle informazioni e di pianificazione del comportamenti, funzionali al contesto (vedi tra gli altri Carver e Scheier, 1983). Alcuni autori (ad esempio Mischel, 1983) descrivono queste strategie "dinamiche» senza sottolineare le differenza interindividuali nella formazione degli scopi. La maggior parte degli scopi sono strettamente connessi a specifici processi cognitivi “imposti» dalla situazione (Wyer, Srull, Gordon, Hartwick, 1982); gli scopi possono inoltre essere «costriuti» dall'individuo nella situazione, di volta in volta diversa. Uno scopo personalmente costruito solitamente ha una caratteristica di "forte rilevanza per il sé» e può rappresentare importanti aspetti del concetto di sé. Gli scopi sono quindi compresi nell'insieme dei sé possibili (Markus, 1983). Markus e Nurius (1986) definiscono infatti i sé possibili come «le componenti cognitive dei desideri, timori, scopi e sensi di pericolo dell'individuo; essi danno un significato, un'organizzazione, una direzione e un carattere di rilevanza per il sé, specifici a queste dinamiche" (p. 954). I sé possibili possono essere gli strumenti che portano a degli intensi e temporanei cambiamenti nella valutazione di sé; essi possono allo stesso modo essere i meccanismi che guidano i cambiamenti duraturi nella concezioni di sé. Se accettiamo la possibilità che i sé possibili siano le componenti abituali del concetto di sé, possiamo altresi accettare l'ipotesi di un concetto di sé composto da vari elementi e multisfaccettato. Quindi i sé possibili costituiscono un concetto di sé complesso e variabile ma al tempo stesso autentico e coerente nel senso che rappresenta i desideri e timori presenti nell'individuo che possono essere realizzati in condizioni sociali appropriate. In questa prospettiva, $i$ sé possibili contribuiscono a determinare la fluidità e malleabilità del sé in quanto vengono attivati in maniera differenziata nelle situazioni sociali e determinano la natura del sé operante (working self-concept). Si può presumere quindi che nel nostro caso i soggetti abbiano fornito un'auto-descrizione attivando un sé possibile in funzione della situazione e che tale sé possibile sia rimasto operante anche in fase di recupero delle informazioni condizionando la rievocazione. 


\section{Referencias}

BELLEZZA, F. S. (1984). "The self as a mnemonic device; The role of internal cues». Journal of Personality and Social Psychology, 47: 506-516.

BOWER, G. H. y GILLIGAN, S. G. (1979). «Remembering information related to one's self". Joumal of Research in Personality, 13: 420-432.

Brown, J. D. (1986). *Evaluations of self and others: Self-enhancement biases in social judgementw. Social Cognition, 4: 353-376.

Carver, C. S. y SCHEIER, M. F. (1983). *A control-theory approach to human behavoir and implications for problems in self-management. . In Kendall, P. C. (ed.) Advances in cognitive-behavioral research and therapy (Vol. 2): 127-194. New York: Academic Press.

EPSTEIN, S. (1983). "The unconscious, the preconscious, and the self-concept». In Suls, J. y Greenwald, A. G. (eds.), Psychological perspectives on the self (Vol. 2): 219-247. Hillsdale, NJ: Erlbaum.

FORZI, M. (1988). "Auto-accrescimento e coerenza: due orientamenti del sé». Reports from Institute of Trieste.

FORZI, M.; KoDILJA, R. y ARCURI, L. (1988). «Self-conception, self-reference and memory performance». (Inviato per la pubb.) European Journal of Personality.

GERGEN, K. J. (1979). «Sé fluido e sé rigido". In Giovannini, D. (a cura di), Identità personale, teoria e ricerca. Bologna: Zanichelli.

GREENWALD, A. G. (1981). *Self and memory*. In Bower, G. H. (ed.). The psychology of learning and motivation (Vol. 15) New York: Academic Press.

Greenwald, A. G. y Pratkanis, A. R. (1984). "The self». In Wyer, R. S. y Srull, T. K. (eds.), Handbook of social cognition (Vol. 3). Hillsdale, NJ: Erlbaum.

HIGGINS, E. T. y MCCANN, C.D. (1984). «Social encoding and subsequent attitudes, impressions, and memory: "Context-driven" and motivational aspects of processing". Journal of Personality and Social Psychology, 47: 26-39.

JONES, S. C. (1973). «Self and interpersonal evaluations: Esteem theories versus consistency theories». Psychological Bulletin, 79: 185-199.

KEENAN, J. M. y BAILLET, S. D. (1980). "Memory for personally and socially significant events". In R. S. Nickerson (ed.), Attention and performance VIII. 651-699. Hillsdale, NJ: Erlbaum.

KiHLSROM, J. F. y CANTOR, N. (1984). "Mental representation of the self". In L. Berkowitz (ed.), Advances in Experimental Social Psychology (Vol. 17). New York: Academic Press.

Kihlstrom, J. F.; Cantor, N.; Albright, J. S.; Chew, B. R.; Klein, S. B y Niedenthal, P. M. (1988). "Information processing and the study of the self». In L. Berkowitz (ed.). Advances in Experimental Social Phychology. (Vol. 21). New York: Academic Press.

KLEIN, S. B. y KIHLSTROM, J. F. (1986). "Elaboration, organization, and the self-reference effect in memory". Joumal of Experimental Psychology: General, 115: 26-38.

KUIPER, N. A. (1981). "Convergent evidence for the self as a prototype: The "Inverted-URT Effect" for the self and other judgements». Personality and Social Psychology Bulletin, 7: 438-443.

KUIPER, N. A. y ROGERS, T. B. (1979). «Encoding of personal information: Self-other differences". Journal of Personality and Social Psychology, 37: 499-514.

LECKY, P. (1945). Self-consistency: $A$ theory of personality. New York: Island Press.

LORD, C. G. (1980). "Schemas and images as memory aids: Two modes of processing social information. Journal of Personality and Social Psychology. 38: 257-269.

MARKUS, $H$. (1977). "Self-schemata and processing information about the self". Journal of Personality and Social Psychology, 35: 63-78.

MARKUS, H. (1983). «Self-knowledge: An expanded view m. Journal of Personality, 51: 543-565.

MARKuS, H. y NURIUS, P. (1986). «Possible selves». American Psychologist, 41: 954-969.

MARKUS, H. y SENTIS, K. (1982). *The self in social information processing*. In Suls, J. (ed.), Psychological perspectives on the self (Vol. 1). Hillsdale, NJ: Erlabaum.

MARKUS, H. y WURF, E. (1987). "The dynamic self-concept: A social psychological perspectivem. Annual Review of Psychology, 38.

McCAUL, K. D. y MAKI, R. H. (1984). «Self-reference versus desirability ratings and memory for traitsw. Journal of Personality and Social Psychology, 47: 953-955.

McGuire, W. J. y McGuire, C. V. (1981). "The spontaneus self-concept as affected by personal distinctivenessn. In M. D. Lynch, A. A. Norem-Hebeisen y K. Gergen (eds.), Self-concept: Advances in theory and research. New York: Ballinger.

McGuire, W. J.; McGuire, C. V. y CHEEver, J. (1986). *The self in society: Effects of social contexts on the sense of the self . British Journal of Social Psychology, 25: 259-270.

McGuire, W. J. y PADAWER-Singer, A. (1976). "Trait salience in the spontaneous self-concept». Journal of Personality and Social Psychology, 33: 743-754.

MISCHEL, W. (1983). «Delay of gratification as process and as person variable in development". In D. Magnusson y V. P. Allen (eds.), Human Development: An interactional perspective. New York: Academic. 
MISCHEL, W. (1984). "Convergences and challenge in the search for consistency". American Psychologist, 39: 351-364.

MORELAND, R. L. y SWEENEY, P. D. (1984). "Self-expectancies and reactions to evaluations of personal performance». Journal of Personality, 52: 156-176.

MORSE, S. y GERGEN, K. J. (1970). "Social comparison, self-consistency, and the concept of the self". Journal of Personality and Social Psychology, 36: 72-81.

ROGERS, T. B. (1981). "A model of the self as an aspect of the human information processing system". In N. Cantor y J. F. Kihlstrom (eds.), Personality, cognition and social interaction. Hillsdale, $\mathrm{NJ}$ : Erlabaum.

ROGERS, T. B.; KUIPER, N. A. y KIRKER, W. S. (1977). "Self-reference and the encoding of personal information". Journal of Personality and Social Psychology, 35: 677-688.

SECORD, P. F. y BACKMAN, C. W. (1965). "An interpersonal approach to personality". In B. Maher (ed.), Progress in experimental personality research (Vol. 2), 91-125. New York: Academic Press.

SHOWERS, C. y CANTOR, N. (1985). «Social cognition: A look at motivated strategiesm. Annual Review of Psychology, 36: 275-305.

SHRAUGER, J. S. (1975). "Responses of evaluation as a function of initial self-perceptions". Psychological Bulletin, 82: 581-596.

SNYDER, M. (1979). "Self-monitoring processes". In L. Berkowitz (ed.), Advanced in Experimental Social Psychology (Vol. 12). New Yokr: Academic Press.

SWANN, W. B., Jr. (1983). «Self-verification: Bringin social reality into harmony with the self". In Suls, J. y Greenwald, A. G. (eds.), Social psychological perspectives on the self (Vol. 2), 33-66. Hillsdale, NJ: Erlbaum.

SwanN, W. B., Jr.; Griffin, J. J., Jr.; Predmore, S. C. y Gaines, B. (1987). «The cognitive-affective crossfire: When self-consistency confronts self-enhancement». Journal of Personality and Social Psychology, 52: 881-889.

WYeR, R. S.; SRULL, T. K.; GORDON, S. E. y HARTWICK, J. (1982). «Effects of processing objectives and the recall of prose material.. Journal of Personality and Social Psychology, 43: 674-688. 